\title{
Ecuaciones alométricas de biomasa aérea para la estimación de los contenidos de carbono en manglares del Caribe Colombiano
}

\author{
Adriana Yepes ${ }^{1}$, Mauricio Zapata ${ }^{1}$, Jhoanata Bolivar ${ }^{1}$, Alejandra Monsalve ${ }^{2}$, Sandra Milena \\ Espinosa $^{2}$, Paula Cristina Sierra-Correa ${ }^{3} \&$ Andrés Sierra $^{1}$ \\ 1. Centro de Investigación en Ecosistemas y Cambio Global Carbono \& Bosques. Calle 51A \# 72-23 Interior 601, \\ Medellín,Colombia; adrianayepes@carbonoybosques.org, mauricio.zapata98@gmail.com, jbolivar@carbonoy- \\ bosques.org, asierrab@carbonoybosques.org \\ 2. Universidad Nacional de Colombia, Sede Medellín. Programa curricular de Ingeniería Forestal. Calle 59A No 63-20 \\ - Núcleo El Volador, Medellín, Colombia; alejamonsalver@gmail.com, smespino@unal.edu.co \\ 3. Instituto de Investigaciones Marinas y Costeras "José Benito Vives de Andreis" - INVEMAR. Calle 25 No. 2-55, \\ Playa Salguero, Santa Marta; paula.sierra@invemar.org.co
}

Recibido 17-II-2015. Corregido 16-XI-2015. Aceptado 15-XII-2015.

\begin{abstract}
Tree above-ground biomass allometries for carbon stocks estimation in the Caribbean mangroves in Colombia. The distribution of carbon in "Blue Carbon" ecosystems such as mangroves is little known, when compared with the highly known terrestrial forests, despite its particular and recognized high productivity and carbon storage capacity. The objective of this study was to analyze the above ground biomass (AGB) of the species Rhizophora mangle and Avicennia germinans from the Marine Protected Area of Distrito de Manejo Integrado (DMI), Cispatá-Tinajones-La Balsa, Caribbean Colombian coast. With official authorization, we harvested and studied 30 individuals of each species, and built allometric models in order to estimate AGB. Our AGB results indicated that the studied mangrove forests of the DMI Colombian Caribbean was of 129.69 $\pm 20.24 \mathrm{Mg} / \mathrm{ha}$, equivalent to $64.85 \pm 10.12 \mathrm{MgC} / \mathrm{ha}$. The DMI has an area of 8570.9 ha in mangrove forests, and we estimated that the total carbon potential stored was about $555795.93 \mathrm{Mg} \mathrm{C}$. The equations generated in this study can be considered as an alternative for the assessment of carbon stocks in AGB of mangrove forests in Colombia; as other available AGB allometric models do not discriminate mangrove forests, despite being particular ecosystems. They can be used for analysis at a more detailed scale and are considered useful to determine the carbon storage potential of mangrove forests, as a country alternative to support forest conservation and emission reduction strategies. In general, the potential of carbon storage from Colombian Caribbean mangrove forests is important and could promote the country leadership of the "blue carbon" stored. Rev. Biol. Trop. 64 (2): 913-926. Epub 2016 June 01.
\end{abstract}

Key words: A. germinans, above ground biomass, allometric equations, carbon, mangroves, $R$. mangle.

La estimación de los contenidos y flujos de carbono en bosques tropicales es, en la actualidad, uno de los principales temas de interés científico, debido a que estos ecosistemas actúan como verdaderos sumideros o fuentes de dióxido de carbono $\left(\mathrm{CO}_{2}\right)$ a la atmósfera (Malhi \& Phillips, 2004; Houghton, 2005; Chave et al., 2005; Brown et al., 2007; Clark, 2007), especialmente cuando de acuerdo con varios estudios, la deforestación tropical ha contribuido con $20 \%$ de las emisiones globales de Gases de Efecto Invernadero (GEI) a la atmósfera en las últimas décadas (IPCC, 2007; Fearnside \& Laurance, 2004; Houghton, 2005; Achard et al., 2007; Olander, Gibbs, Steininger, Swenson, \& Murray, 2008; GOFC-GOLD, 2009; Malhi et al., 2009). No obstante, uno de los principales desafíos que persisten, a nivel mundial, es lograr cuantificar la deforestación y las reservas de carbono de los bosques de manera precisa; identificar su localización y llegar a predecir a través del monitoreo, su 
comportamiento en el tiempo (GOFC-GOLD, 2009; Gibbs, Brown, Niles, \& Foley, 2007). En este sentido, las ecuaciones alométricas son una herramienta esencial para determinar el almacenamiento y los flujos de carbono (Chave et al., 2005; IPCC, 2007) en los ecosistemas forestales. Un gran número de ecuaciones alométricas han sido publicadas en la literatura especializada desde hace décadas (Brown, 1997; West, Brown, \& Enquist, 1999; Baker et al., 2004; Chave et al., 2005; Sierra et al., 2007; Zianis, 2008; Navar, 2009; Álvarez et al., 2012). Sin embargo, pocos de estos estudios se han concentrado en los ecosistemas boscosos de manglar, que son particulares y únicos desde el punto de vista ecológico, y que por lo tanto, deberían de tener una alometría especial.

Los manglares se encuentran entre los ecosistemas más productivos del planeta, con una producción media de $2.5 \mathrm{gC} / \mathrm{m}^{2} /$ día (Jennerjahn \& Ittekkot, 2002), y por ello son bien conocidos por su alta acumulación de carbono, con reportes de de almacenes superiores a los $1000 \mathrm{MgC} / \mathrm{ha}$ (Donato et al., 2011). Esta característica los convierte en ecosistemas claves para mitigar el calentamiento global, y esto a pesar de que representan menos de $1 \%$ de los bosques tropicales, y menos de $0.4 \%$ de los bosques del mundo (Spaulding, Blasco, \& Field 1997). En la actualidad, los bosques de manglar presentan una de las tasas de deforestación más altas del mundo (Duke et al., 2007), debido a procesos como la urbanización, la explotación y el aumento del nivel del mar (Rivera-Monroy et al., 2004). La conversión de bosque de manglar a otros usos, ha provocado la pérdida de más de un tercio de ellos en los últimos 20 a 50 años (Alongi, 2002); dentro de las principales causas de la reducción de los manglares están: la expansión de la agricultura, las prácticas acuícolas no sustentables, el desarrollo de infraestructura costera, las alteraciones localizadas que generan pérdidas de la biomasa, y la consecuente descomposición de materia orgánica, que además, provoca la liberación de grandes cantidades de $\mathrm{CO}_{2}$ a la atmósfera (Lovelock, Ruess, \& Feller, 2011). Dadas las grandes existencias de carbono en los manglares, las emisiones resultantes de gases de efecto invernadero (GEI) son altas, y por tanto, la protección y restauración de los manglares, constituyen una estrategia importante para la mitigación del cambio climático (Adame et al., 2013).

A nivel mundial, los bosques de manglar son generalmente subestimados, sobreexplotados y mal administrados (Ewel, Bourgeois, Cole, \& Zheng, 1998). No obstante, su importancia para los seres humanos, la fauna y el equilibrio global del carbono es fundamental (Walters et al., 2008). El objetivo de este estudio fue i) generar modelos alométricos para estimar la biomasa aérea y el contenido de carbono de los bosques de manglar localizados en el caribe colombiano y ii) estimar la biomasa aérea y carbono en el área de estudio para determinar su potencial en estrategias de mitigación al cambio climático.

\section{MATERIALES Y MÉTODOS}

Área de estudio: El estudio se desarrolló en la Bahía de Cispatá, localizada en el Caribe continental colombiano departamento de Córdoba entre los municipios de San Antero, San Bernardo del Viento y Santa Cruz de Lorica. El área está enmarcada entre las coordenadas geográficas $09^{\circ} 24^{\prime} 0$ " - $09^{\circ} 20^{\prime} 0$ " N y 7549'30" - 75'54'30" W. La cobertura en bosques de manglar es de 8570.9 ha, de las cuales 4163 ha son susceptibles de aprovechamiento forestal (CVS \& INVEMAR., 2010). La elevación promedio del sitio es 5 m.s.n.m, y el régimen climático en la zona es unimodal - biestacional, con una estación seca bien definida entre diciembre y marzo, y una húmeda, entre abril y noviembre (CVS \& IAvH, 2006). La temperatura media mensual oscila entre 26.7 y $28.6{ }^{\circ} \mathrm{C}$ (Sánchez-Páez, Ulloa-Delgado, Tavera Escobar, \& Gil Torres, 2005); la evaporación potencial promedio es de $1826 \mathrm{~mm} / \mathrm{año}$, y la precipitación anual promedio es de 1425 mm (Sánchez-Páez, Ulloa-Delgado, \& TaveraEscobar, 2004). Se puede considerar como una de las áreas del litoral Caribe colombiano más dinámica, porque según Robertson y Chaparro 
(1998), en los últimos siglos el delta del río Sinú ha migrado cuatro veces y ha tomado el nombre de las áreas donde desemboca el río: Los Venados hasta 1762, Mestizos: 1762-1849, Cispatá: 1849-1938 y Tinajones: desde 1938 a la actualidad (Sánchez-Páez et al., 2005). De acuerdo con el sistema de clasificación del Holdridge, Grenke, Hatheway, Liang y Tosi (1971), el área corresponde a las zonas de vida bosque húmedo y muy húmedo tropical (bh-T y bmh-T). En el área, se distinguen otros usos del suelo asociados a los bosques de manglar, como acuacultura, helechales, bosques secos humedales halófilos, playones aluviales y fluviomarinos.

Los bosques de mangle de la Bahía de Cispatá están representados por cuatro especies: Avicennia germinans (L.) Stearn, Rhizophora mangle (L.), Laguncularia racemosa (L.) C.F. Gaertn., y Pelliciera rhizophorae Planch. \& Triana. Las especies Avicennia germinans y Rhizophora mangle presentan los mayores Índices de Valor de Importancia (IVI) (Agudelo et al., 2014), y por tanto, los árboles seleccionados para la construcción de las ecuaciones alométricas correspondieron a estas dos especies.

Selección de la muestra: Además del IVI, se revisó las tendencias de las distribuciones diamétricas para estas especies en 23 parcelas permanentes de $500 \mathrm{~m}^{2}(20 \times 25 \mathrm{~m})$ establecidas en la bahía de Cispatá entre febrero y marzo de 2012 (Agudelo et al., 2014). Se definieron rangos diamétricos de $10 \mathrm{~cm}$ a partir de $2.5 \mathrm{~cm}$. A los rangos con mayor frecuencia se les asignó una mayor cantidad de árboles para la muestra y a los menos frecuentes menor número de individuos.

Trabajo de campo: Se cosecharon en total 60 árboles: $30 \mathrm{~A}$. germinans y 30 de $R$. mangle siguiendo los protocolos existentes para ello (Rügnitz, Chacón, \& Porro, 2009; Yepes et al., 2011). La cosecha de dichos individuos estuvo amparada por la resolución No. 1.5977 del 14 de febrero 2012, emitida por la Corporación Autónoma Regional de los Valles del Sinú y del San Jorge - CVS, Autoridad Ambiental con jurisdicción en el área de estudio. A cada árbol seleccionado se le registró las coordenadas de su ubicación (se empleó un GPS map 60Scx Garmin). Se midió el diámetro normal (DAP) (diámetro a $1.30 \mathrm{~m}$ de altura o $30 \mathrm{~cm}$ por encima de la última raíz aérea cuando la hubo, y el diámetro en la base $(\mathrm{Db})$. Se empleó la cinta métrica (precisión de $1.0 \mathrm{~mm}$ ) para diámetros mayores o iguales a $10 \mathrm{~cm}$, y pie de rey para los diámetros menores a $10 \mathrm{~cm}$ (precisión $0.1 \mathrm{~mm}$ ). Se midió la altura de copa (Hc) y la altura total (Ht) antes del apeo (con clinómetro SUNNTO, precisión $0.5^{\circ}$ ). En el árbol apeado, siguiendo el método de Smalian reiterado (Lema, 2003), se realizaron mediciones del diámetro del fuste cada décimo de la longitud para hallar su volumen con corteza. Debido a las dificultades presentadas para pesar algunas ramas y raíces aéreas, se recurrió igualmente al método de cubicación. Cada árbol de $R$. mangle y $A$. germinans fue pesado por componentes, separando: hojas-flores-frutos, ramas, raíces aéreasfuste. Cada componente se pesó en verde (se empleó una báscula digital con capacidad máxima de $200 \mathrm{~kg}$ y precisión de $0.1 \mathrm{~kg}$ ) y se tomaron 60 muestras de aproximadamente 300 g para determinaciones de contenido de humedad. Para las determinaciones de densidad de la madera, se tomaron tres muestras en forma de rodaja de cada árbol: en la base, el medio y la parte alta del fuste. Las muestras se empacaron en bolsas plásticas debidamente rotuladas.

Fase de laboratorio: En atención a las recomendaciones de Picard, Saint-André y Henry (2012), el procesamiento de las muestras se realizó en un lapso de tiempo no mayor a seis horas después de recolectadas. Se determinó el volumen de las muestras de madera a partir de mediciones de área transversal y grosor de las rodajas. Cuando las muestras de madera presentaron un diámetro mayor a $20 \mathrm{~cm}$ se tomó una sub-muestra del $20 \%$ de la muestra. Las muestras de cada componente por árbol fueron secadas en hornos a una temperatura de $60{ }^{\circ} \mathrm{C}$ para el caso de las hojas-flores-frutos, y a 105 ${ }^{\circ} \mathrm{C}$ para los componentes maderables ramas, raíces y rodajas, hasta alcanzar peso constante. 
Posteriormente las muestras secas se pesaron en balanza digital con capacidad máxima de 4 $240 \mathrm{~g}$ y precisión de $0.01 \mathrm{~g}$.

Cálculo de biomasa por componente: La biomasa para los componentes hojas-floresfrutos, raíces aéreas y ramas de cada árbol se calculó a partir de la siguiente relación (1):

$$
\mathrm{B}_{\mathrm{ti}}=\left[\mathrm{PS}_{\mathrm{ti}} / \mathrm{PV}_{\mathrm{ti}}\right] \times \mathrm{PV}_{\mathrm{ti}}
$$

Donde, $\mathrm{B}_{\mathrm{ti}}$ es la biomasa o total para el componente $\mathrm{t}$ del árbol i en gramos $(\mathrm{g})$; $\mathrm{PS}_{\mathrm{ti}} \mathrm{I}$ $\mathrm{PV}_{\mathrm{ti}}$ es la relación peso seco - peso verde de las muestras en el componente $\mathrm{t}$ del árbol $\mathrm{i}$; $\mathrm{PV}_{\mathrm{ti}}$ es el peso verde del componente $\mathrm{t}$ en el árbol i obtenido de campo en gramos (g). Para el componente fuste, se determinó primero la densidad anhidra promedia de las tres muestras de madera de cada árbol $\left(\mathrm{g} / \mathrm{cm}^{3}\right)$. Luego, la biomasa real de cada árbol, se halló multiplicando el volumen de cubicación de cada árbol por la densidad anhidra promedia de la muestra. Finalmente, la biomasa aérea total de cada árbol correspondió a la suma de la biomasa encontrada en cada componente, es decir, la biomasa de hojas-flores-frutos, más la biomasa de ramas, y la biomasa de raíces aéreas-fuste.

Se probaron varias formas funcionales de modelos alométricos para explicar la biomasa aérea total en función de variables independientes como DAP, Ht, Db y Hc o combinaciones de éstas. Se empleó la transformación logarítmica, para convertir los modelos no lineales a modelos lineales, y posteriormente, proceder a la estimación de los parámetros mediante el método de mínimos cuadrados ordinarios por regresiones simples. La aptitud o capacidad del modelo fue determinada con pruebas matemáticas y gráficas que evaluaran el cumplimiento de los postulados que sustentan la regresión lineal. La linealidad de la función se comprobó a partir del análisis gráfico de residuales. Para detectar la existencia de auto-correlación de errores se utilizó el estadístico de DurbinWatson (Canavos, 1988); así mismo, para detectar heterocedasticidad se utilizó la prueba de Goldfeld-Quandt (Lema, 2003). La normalidad en los términos del error se determinó a partir de la prueba de Shapiro Wilks (Cochran, 2000). Como criterios de selección estadísticos se utilizaron el coeficiente de determinación ajustado $\left(R a^{2}\right)$, y el Cuadrado Medio del Error (CME). Para cada una de las especies objeto de estudio ( $R$. mangle y $A$. germinans), y para el bosque en general se evaluaron los siguientes tres modelos:

$$
\begin{gathered}
\mathrm{B}_{\mathrm{i}}=\mathrm{a}+\mathrm{b}^{*} \mathrm{X}_{\mathrm{i}}+\mathrm{e}_{\mathrm{i}} \\
\operatorname{Ln}\left(B_{i}\right)=a+b^{*} X_{i}+e_{i} \\
\operatorname{Ln}\left(B_{i}\right)=a+b^{*} \ln \left(X_{i}\right)+e_{i}
\end{gathered}
$$

La variables independientes $\left(X_{i}\right)$ utilizadas en estos modelos fueron DAP, DAP ${ }^{2}, \mathrm{DAP}^{*} \mathrm{Ht}$, $\mathrm{DAP}^{2 *} \mathrm{Ht},(\mathrm{DAP} * \mathrm{Ht})^{2}, \mathrm{AB}$ (área basal en $\mathrm{m}^{2} /$ ha), $\mathrm{AB}^{2}, \mathrm{y} \mathrm{AB}^{*} \mathrm{Ht}$, para un total de 24 modelos; cada una de estas variables, correspondió a las medidas para cada árbol cosechado, es decir, se trabajó con un $\mathrm{n}=30$. Todos los análisis estadísticos se realizaron con la versión 2.9.0 del software $\mathrm{R}$ ( $\mathrm{R}$ Development Core Team, 2010).

Estimación de biomasa aérea total y contenido de carbono: El modelo seleccionado (aquel que proporcionó mejores estadísticos), se utilizó para la estimación de la biomasa aérea de todos los individuos muestreados en cada una de las 23 parcelas permanentes establecidas. El error de muestreo asociado a estas parcelas fue del $9 \%$ (para la variable $\mathrm{AB}$ ) a un nivel de significancia del $95 \%$. El muestreo utilizado fue un diseño aleatorio, las parcelas fueron de $500 \mathrm{~m}^{2}(20 \times 25 \mathrm{~m})$ y se subdividieron en 20 cuadrantes de $5 \times 5 \mathrm{~m}$. En cada parcela, se midió y clasificaron los árboles en fustales, cuando el diámetro normal -DAP- (medido a $1.30 \mathrm{~m}$ de altura sobre el nivel del suelo) fue mayor de $\geq 2.5 \mathrm{~cm}$; latizales, cuando la altura estuvo entre $>1.0 \mathrm{~m}$ y DAP $<2.5 \mathrm{~cm}, \mathrm{y}$ brinzales, es decir, plántulas $\leq 1.0 \mathrm{~m}$ de altura (Agudelo et al., 2014).

La biomasa aérea fue estimada para todas las especies vegetales, incluyendo otras plantas de otras especies diferentes a $A$. germinans y $R$. mangle dentro de las parcelas. Teniendo 
en cuenta los resultados de la caracterización estructural y composición florística de parcelas permanentes en bosques de manglar de la bahía de Cispatá (Córdoba) (Agudelo et al., 2014), en el área de estudio al parecer existe diferencia entre dos tipos de manglar, uno de ellos dominando por $A$. germinans y el otro por $R$. mangle. Basados en la clasificación de Lugo y Snedaker (1974), en la dominancia de cada una de las especies y en el comportamiento de las variables ambientales asociadas. Agudelo et al., (2014) clasifican estos tipos como manglares de cuenca para el caso de aquel dominado para A. germinans, y de borde y/o ribereño para el tipo de manglar dominado por $R$. mangle.

Los contenidos de carbono fueron estimados utilizando un factor de conversión de biomasa a carbono de 0.50 , tal como lo recomiendan varios autores (McDicken, 1997; Fearnside, Lashof, \& Moura-Costa, 1999; Clark et al., 2001; Chave et al., 2005; Aragão et al., 2009).

\section{RESULTADOS}

Para la especie $R$. mangle, $60 \%$ de los árboles cosechados correspondieron al rango entre 2.5 y $12.5 \mathrm{~cm}$ de diámetro normal, $23 \%$ al rango entre 12.5 y $22.5 \mathrm{~cm}, 10 \%$ entre 22.5 y $32.5 \mathrm{~cm}$, y $7 \%$ entre 32.5 y $42.5 \mathrm{~cm}$. Para la especie $A$. germinans $57 \%$ de los árboles cosechados correspondieron al rango entre 2.5 y $12.5 \mathrm{~cm}$ de diámetro normal, $20 \%$ al rango entre 12.5 y $22.5 \mathrm{~cm}, 13 \%$ entre 22.5 y 32.5 $\mathrm{cm}, 3 \%$ entre 32.5 y $42.5 \mathrm{~cm}, 3 \%$ entre 42.5 y $52.5 \mathrm{~cm}$, y $3 \%$ entre 52.5 y $62.5 \mathrm{~cm}$. Las distribuciones diamétricas de $R$. mangle y $A$. germinans siguieron la forma de J-invertida, característica de comunidades con estructura poblacional estable (Fig. 1).

Variación en los datos de biomasa aérea tomados en campo: El componente de hojasflores-frutos, fue el que presentó menor proporción respecto a la biomasa aérea total, éste varió de 2.8 a $5.6 \%$ para la especie $A$. germinans, y de 2.0 a $8.4 \%$ para $R$. mangle. Las proporciones más altas de este componente

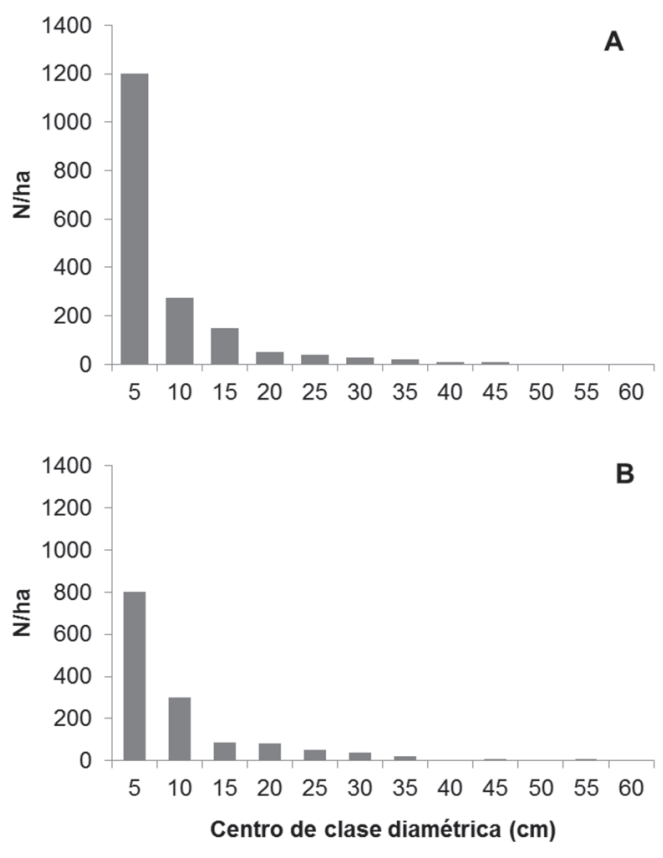

Fig. 1. (A). Distribución diamétrica de $R$. mangle en la Bahía de Cispatá. (B). Distribución diamétrica de $A$. germinans en la Bahía de Cispatá.

Fig. 1. (A). Diameter distribution of R. mangle in the Bay of Cispatá. (B). Diameter distribution of A. germinans in the Bay of Cispatá.

tendieron a presentarse en los individuos más pequeños para ambas especies. En $A$. germinans la proporción de biomasa de ramas presentó los menores valores en los individuos pequeños, mientras que la proporción de biomasa del fuste fue menor en los individuos de mayor tamaño. En $R$. mangle las proporciones de biomasa del fuste y ramas presentaron una distribución heterogénea sin ninguna tendencia clara. Para ambas especies se observó que la proporción de biomasa de ramas puede ser mayor a la proporción de biomasa del fuste en los individuos mayores. El porcentaje de biomasa de raíces aéreas en $R$. mangle presentó una distribución homogénea de $24.7 \pm$ $4.0 \%$, con una representación de hasta 32.6 $\%$ de la biomasa aérea total. En general, la proporción de los componentes hojas, flores, frutos y ramas fue similar para las especies A. germinans y R.mangle (3.9 y $5.1 \%$, 
respectivamente). No obstante, el porcentaje del fuste en $R$. mangle fue significativamente menor al de $A$. germinans, con 43.3 y $64.4 \%$, respectivamente (Cuadro 1). El porcentaje faltante en $R$. mangle estuvo dado por la biomasa de raíces aéreas, componente que no estuvo presente en $A$. germinans.

Ecuaciones de biomasa: Los modelos i. $\left(B_{i}=a+b * X_{i}+e_{i}\right)$ y ii. $\left(\operatorname{Ln}\left(B_{i}\right)=a+b * X_{i}+e_{i}\right)$ fueron descartados ya que no cumplieron con los supuestos de linealidad, heterocedasticidad ni normalidad para ninguna de las variables evaluadas (Cuadro 2). El modelo iii. $\left(\operatorname{Ln}\left(B_{i}=a+b * \ln \left(X_{i}\right)+e_{i}\right)\right.$ presentó un buen ajuste para las ocho variables estudiadas. En el cuadro 2 se observan las ecuaciones de biomasa aérea general y para las dos especies de manglar objeto de estudio que presentaron mejor ajuste. En los tres casos se escogió el modelo 1, debido a que éstos presentaron el mayor valor de $R a^{2}$ y de $F$ y el menor CME, además las pruebas de comparación entre los datos observados y estimados demostraron que no existe diferencia estadísticamente significativa entre los valores de biomasa observados y estimados a un nivel de confianza del $95 \%$. Adicionalmente, la prueba Goldfeld-Quandt mostró que ninguna de las ecuaciones seleccionadas presenta problemas de heterocedasticidad, y según la prueba de Durbin Watson no existe auto-correlación de errores en estos modelos. La prueba de distancia de Cook, mostró que los datos no presentan observaciones remotas para las ecuaciones seleccionadas $(\mathrm{Di}>1)$.

Biomasa aérea total y contenido de carbono: La biomasa aérea se estimó a partir de la ecuación general seleccionada $\operatorname{Ln}\left(B_{i}=a+b^{*} \ln \left(X_{i}\right)+e_{i}\right)$, ya que ésta no presentó diferencias estadísticamente significativas entre los valores de biomasa observados para cada una de las especies y los estimados a un nivel de confianza del $95 \%$. Del mismo modo se asumió que esta ecuación es un buen estimador de la biomasa aérea para las especies Pelliciera rhizophorae Planch. \& Triana y Laguncularia racemosa (L.) C.F. Gaertn., las cuales no fueron incluidas en la construcción de las ecuaciones debido al bajo índice de valor de importancia (IVI) que presentaron estas especies para la zona de estudio, de $5 \%$ y $35 \%$, respectivamente. Para conocer si la especie dominante influía sobre los valores de biomasa aérea, se realizó un análisis de varianza (ANAVA) entre los grupos identificados. Éste mostró que no existe diferencia significativa entre los grupos con un nivel de confianza del $95 \%$, indicando que la biomasa área de los bosques de manglar de la bahía de Cispatá no se ve afectada por la especie dominante. De esta manera, la biomasa aérea total de los bosques de manglar en el DMI es de $129.69 \pm$ $20.24 \mathrm{Mg} /$ ha (Cuadro 3). Teniendo en cuenta que el DMI presenta un área de 8570.9 ha en manglar, se estimó que la biomasa aérea total de este ecosistema en el área de estudio, es de 1 $111591.87 \mathrm{Mg}$. Por su parte, el contenido total de carbono almacenado en la biomasa aérea es de $64.85 \pm 10.12 \mathrm{MgC} / \mathrm{ha}$, lo que corresponde a $555795.93 \mathrm{Mg}$.

\section{DISCUSIÓN}

Numerosos estudios que han usado modelos de regresión para determinar la biomasa aérea de especies de manglar reportan que el DAP solo, o asociado con la altura (Ht), son las variables más importantes y relevantes en esta estimación (Fromard et al., 1998; Soares \& Schaeffer-Novelli, 2005). En el presente estudio se encontró que el modelo logarítmico con el diámetro normal (DAP) como variable dependiente, estima eficientemente la biomasa total de los bosques de manglar de la bahía de Cispatá. Este modelo utilizado en bosques tropicales naturales, presenta muchas ventajas dada la facilidad de aplicación. En relación con los valores de biomasa aérea obtenidos, algunos estudios han sugerido que hay diferencias específicas entre las especies en la proporción de los componentes de la biomasa, especialmente en las especies que difieren en la arquitectura (Alongi, 2009). En este estudio, la variación encontrada en la proporción de los componentes de biomasa aérea de $R$. mangle y 


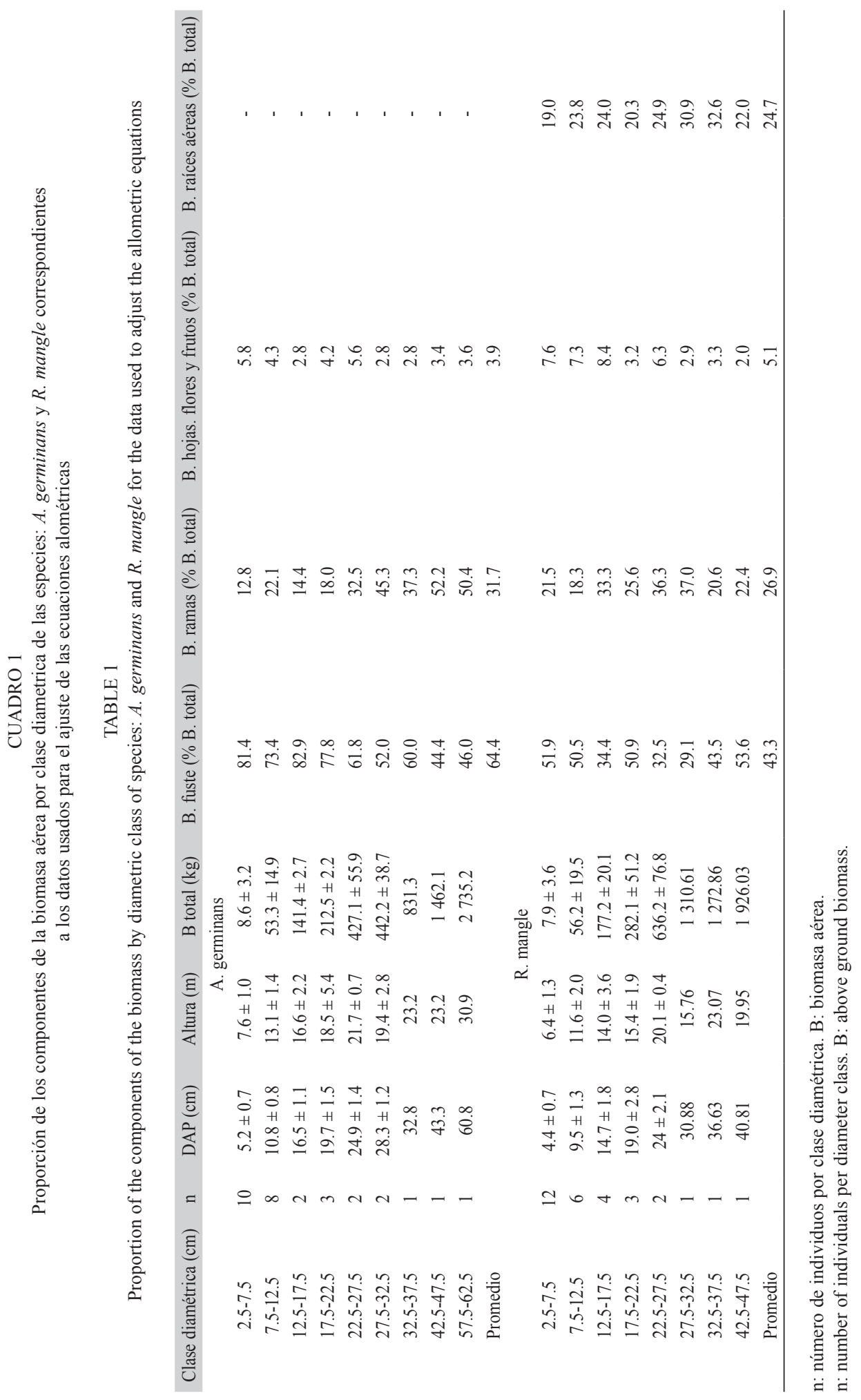

(c) (i) Rev. Biol. Trop. (Int. J. Trop. Biol. ISSN-0034-7744) Vol. 64 (2): 913-926, June 2016 
CUADRO 2

Estadísticos de regresión para ecuaciones de biomasa aérea

TABLE 2

Statistical regression equations for above ground biomass

\begin{tabular}{|c|c|c|c|c|}
\hline Modelo & $\mathrm{n}$ & $\mathrm{Ra}^{2}$ & CME & $\mathrm{F}$ \\
\hline \multicolumn{5}{|l|}{ Avicennia germinans } \\
\hline $\mathrm{B}=-354.18+39.72 \mathrm{DAP}$ & 30 & 0.86 & 44983 & 173 \\
\hline $\ln B=2.06+0.13 \mathrm{DAP}$ & 30 & 0.82 & 0.64 & 131 \\
\hline $\ln B=-1.96+2.45 \ln \mathrm{DAP}$ & 30 & $0.99 *$ & 0.03 & 3039 \\
\hline $\ln B=-1.96+1.23 \ln \operatorname{laP}^{2}$ & 30 & 0.99 & 0.03 & 3039 \\
\hline $\operatorname{lnB}=-3.52+1.52 \operatorname{lnDAP} * \mathrm{Ht}$ & 30 & $0.98^{*}$ & 0.08 & 1304 \\
\hline $\ln B=-2.95+0.94 \ln \mathrm{DAP}^{2} * \mathrm{Ht}$ & 30 & $0.99 *$ & 0.04 & 2245 \\
\hline $\ln B=-3.53+0.76 \ln (\mathrm{DAP} * \mathrm{Ht})^{2}$ & 30 & 0.98 & 0.08 & 1304 \\
\hline $\ln B=9.62+1.23 \ln A B$ & 30 & 0.99 & 0.03 & 2981 \\
\hline $\ln B=9.62+0.61 \ln A^{2}$ & 30 & 0.99 & 0.03 & 2981 \\
\hline $\ln \mathrm{B}=5.94+0.94 \ln \mathrm{AB} * \mathrm{Ht}$ & 30 & 0.99 & 0.05 & 2233 \\
\hline \multicolumn{5}{|l|}{ Rhizophora mangle } \\
\hline $\mathrm{B}=-282.97+42.40 \mathrm{DAP}$ & 30 & 0.86 & 29580 & 183 \\
\hline $\operatorname{lnB}=1.54+0.19 \mathrm{DAP}$ & 30 & 0.85 & 0.66 & 160 \\
\hline $\ln B=-1.91+2.59 \operatorname{lnDAP}$ & 30 & $0.99 *$ & 0.04 & 3273 \\
\hline $\operatorname{lnB}=-1.91+1.30 \operatorname{lnDAP}{ }^{2}$ & 30 & 0.99 & 0.04 & 3273 \\
\hline $\operatorname{lnB}=-3.16+1.55 \ln \mathrm{DAP} * \mathrm{Ht}$ & 30 & $0.96^{*}$ & 0.16 & 748 \\
\hline $\ln B=-2.73+0.98 \ln D A P^{2} * \mathrm{Ht}$ & 30 & $0.98 *$ & 0.09 & 1398.30 \\
\hline $\ln B=-3.16+0.77 \ln (\mathrm{DAP} * \mathrm{Ht})^{2}$ & 30 & 0.96 & 0.16 & 748 \\
\hline $\ln B=10.35+1.30 \ln A B$ & 30 & 0.99 & 0.04 & 3285 \\
\hline $\ln \mathrm{B}=10.35+0.65 \ln \mathrm{AB}^{2}$ & 30 & 0.99 & 0.04 & 3285 \\
\hline $\ln B=6.48+0.98 \ln A B * H t$ & 30 & 0.98 & 0.09 & 1400 \\
\hline \multicolumn{5}{|l|}{ General } \\
\hline $\ln \mathrm{B}=-1.86+2.49 \operatorname{lnDAP}$ & 60 & $0.98 *$ & 0.16 & 2961 \\
\hline $\ln B=-1.86+1.24 \operatorname{lnDAP} 2$ & 60 & 0.98 & 0.16 & 2961 \\
\hline $\operatorname{lnB}=-3.18+1.49 \operatorname{lnDAP} * \mathrm{Ht}$ & 60 & $0.95^{*}$ & 0.37 & 1209.70 \\
\hline $\ln B=-2.72+0.94 \operatorname{lnDAP}{ }^{2} * \mathrm{Ht}$ & 60 & $0.97 *$ & 0.25 & 1844 \\
\hline $\ln B=-3.18+0.75 \operatorname{lnDAP} 2 * H t$ & 60 & 0.95 & 0.37 & 1210 \\
\hline $\ln B=9.89+1.24 \ln A B$ & 60 & 0.98 & 0.16 & 2927 \\
\hline $\ln B=9.89+0.62 \ln A^{2}$ & 60 & 0.98 & 0.16 & 2927 \\
\hline $\ln B=6.17+0.94 \ln A B * H t$ & 60 & 0.97 & 0.25 & 1842 \\
\hline
\end{tabular}

$\ln =$ logaritmo natural; $\mathrm{B}=$ biomasa aérea $(\mathrm{kg}) ; \mathrm{DAP}=$ diámetro normal $(\mathrm{cm}) ; \mathrm{H}=$ altura total $(\mathrm{m}) ; \mathrm{n}=$ número de observaciones; $\mathrm{R}_{\mathrm{a}}{ }^{2}=$ coeficiente de determinación ajustado; $\mathrm{CME}=$ cuadrado medio del error; $\mathrm{F}=$ valor del estadístico $\mathrm{F}$ = calculado.

$\mathrm{ln}=$ natural logarithm; $\mathrm{B}=$ above ground biomass $(\mathrm{kg}) ; \mathrm{DAP}=$ average diameter $(\mathrm{cm}) ; \mathrm{H}=$ total height $(\mathrm{m}) ; \mathrm{n}=$ number of observations; $\mathrm{R}_{\mathrm{a}}{ }^{2}=$ adjusted coefficient of determination; $\mathrm{CME}=$ mean square error; $\mathrm{F}=\mathrm{F}$-statistic calculated.

* Modelos seleccionados/Selected model. 
CUADRO 3

Carbono y biomasa aérea reportados para diferentes bosques de manglar

TABLE 3

Carbon and above ground biomass reported for different mangrove forests

\begin{tabular}{|c|c|c|c|c|c|}
\hline Ubicación & Tipo de bosque & Especie dominante & $\begin{array}{c}\text { Biomasa } \\
\text { aérea }(\mathrm{Mg} / \mathrm{ha})\end{array}$ & $\begin{array}{l}\text { Carbono } \\
\text { (Mg/ha) }\end{array}$ & Fuente \\
\hline $\begin{array}{l}\text { Bahía de Cispatá. } \\
\text { Colombia }\end{array}$ & Manglar/estuario & R. mangle & 129.69 & 64.85 & Presente estudio \\
\hline $\begin{array}{l}\text { Sian Ka'an. Península de } \\
\text { Yucatán. México }\end{array}$ & $\begin{array}{l}\text { Manglar alto } \\
(>5 \mathrm{~m})\end{array}$ & L. racemosa & 176.2 & 84.6 & $\begin{array}{l}\text { Adame et al., } \\
\text { (2013) }\end{array}$ \\
\hline $\begin{array}{l}\text { Sian Ka'an. Península de } \\
\text { Yucatán. México }\end{array}$ & $\begin{array}{l}\text { Manglar alto } \\
(>5 \mathrm{~m})\end{array}$ & R. mangle & 144.9 & 69.6 & $\begin{array}{l}\text { Adame et al., } \\
\text { (2013) }\end{array}$ \\
\hline $\begin{array}{l}\text { Sian Ka'an. Península de } \\
\text { Yucatán. México }\end{array}$ & $\begin{array}{l}\text { Manglar mediano } \\
(3-5 \mathrm{~m})\end{array}$ & R. mangle & 114.2 & 54.8 & $\begin{array}{l}\text { Adame et al., } \\
\text { (2013) }\end{array}$ \\
\hline $\begin{array}{l}\text { Sian Ka'an. Península de } \\
\text { Yucatán. México }\end{array}$ & $\begin{array}{l}\text { Manglar enano } \\
(<1.5 \mathrm{~m})\end{array}$ & R. mangle & 5.3 & 2.5 & $\begin{array}{l}\text { Adame et al., } \\
\text { (2013) }\end{array}$ \\
\hline Sundarbans. India & Manglar/estuario & A. marina & 93.7 & 49.5 & $\begin{array}{l}\text { Ray et al., } \\
(2010)\end{array}$ \\
\hline Indo-Pacífico & Manglar/estuario & $\begin{array}{l}\text { Bruguiera. Rhizophora y } \\
\text { Sonneratia }\end{array}$ & & 101.7 & $\begin{array}{l}\text { Donato et al., } \\
\text { (2011) }\end{array}$ \\
\hline Indo-Pacífico & Manglar oceánico & $\begin{array}{l}\text { Bruguiera. Rhizophora y } \\
\text { Sonneratia }\end{array}$ & & 197.6 & $\begin{array}{l}\text { Donato et al., } \\
\text { (2011) }\end{array}$ \\
\hline Guyana Francesa & $\begin{array}{l}\text { Manglar pionero } \\
\text { (2-3 años) }\end{array}$ & L. racemosa & 31.5 & & $\begin{array}{l}\text { Fromard et al., } \\
\text { (1998) }\end{array}$ \\
\hline Guyana Francesa & $\begin{array}{l}\text { Manglar maduro } \\
\text { (50 años) }\end{array}$ & A. germinans & 180 & & $\begin{array}{l}\text { Fromard et al., } \\
\text { (1998) }\end{array}$ \\
\hline Guyana Francesa & $\begin{array}{l}\text { Manglar maduro } \\
(60-70 \text { años })\end{array}$ & A. germinans, $R$. mangle & 315.0 & & $\begin{array}{l}\text { Fromard et al., } \\
(1998)\end{array}$ \\
\hline
\end{tabular}

A. germinans, especialmente en el componente fuste, se debió en efecto a la diferencia en la arquitectura de estas especies. Específicamente, la especie del género Rhizophora tuvo una proporción considerable de carbono en las raíces aéreas.

Según algunos estudios, la biomasa aérea puede oscilar entre 100 y $200 \mathrm{Mg} /$ ha a nivel pantropical (Lacher, 1977), rango en el cual se encuentra el valor estimado en el presente estudio (129.63 Mg/ha). No obstante, también es posible encontrar valores más altos o más bajos, como los que se presentan en el continente Africano, donde la biomasa aérea de los manglares puede variar entre $72 \mathrm{Mg} / \mathrm{ha}$ y 207 $\mathrm{Mg} /$ ha (Fatoyinbo, Simard, Washington-Allen, \& Shugart, 2008; Fatoyinbo \& Simard, 2013), o en manglares de Indonesia y Malasia, que presentan biomasa aérea de más de $300 \mathrm{Mg}$ / ha (Ong, Gong, \& Wong, 1981; Komiyama,
Moriya, Prawiroatmodjo, Toma, \& Ogino, 1988). En América, se han reportado valores de biomasa aérea de $315.0 \mathrm{Mg} /$ ha para los bosques maduros de la Guyana Francesa (Fromard et al., 1998) y de $5.3 \mathrm{Mg} /$ ha para los bosques enanos de la península de Yucatán, México (Adame et al., 2013). En general, se cree que la productividad forestal es más alta cerca del Ecuador, y que disminuye con el aumento de la latitud. En una revisión de 43 trabajos sobre la estructura, biomasa y la hojarasca de los bosques de manglar de todo el mundo (Saenger \& Snedaker, 1993), se concluyó que las alturas de los árboles de mangle, la biomasa aérea y la hojarasca, siguen este patrón de distribución.

Otras investigaciones han documentado que las diferencias en los valores de biomasa aérea de los bosques de manglar están relacionadas con factores ecológicos que limitan el desarrollo de los manglares (Fromard et al., 
1998). Por ejemplo, los bajos valores de biomasa aérea que se presentan en los manglares enanos del México, se deben a la existencia de condiciones micro-climáticas extremas donde éstos se localizan, como son la alta salinidad que limita el crecimiento de los manglares. Por otro lado, el alto valor de biomasa aérea reportada para los manglares maduros de la Guyana Francesa o los de Indonesia, indica que éstos no presentan ningún factor ecológico que pueda limitar su desarrollo (Fromard et al., 1998).

El contenido de carbono almacenado en la biomasa aérea que se ha reportado para los diferentes bosques de manglar en el trópico muestra marcadas diferencias. Las mayores cantidades de carbono (c.a $197.6 \mathrm{Mg} / \mathrm{ha}$ ) han sido reportadas para los manglares oceánicos del Indo-Pacífico (Donato et al., 2011), mientras que los menores valores $(2.5 \mathrm{Mg}$ / ha), se reportan para los manglares enanos en la península de Yucatán en México (Adame et al., 2013). Las grandes variaciones en el contenido de carbono de los manglares se deben a las diferencias estructurales y florísticas entre los bosques (Adame et al., 2013), de manera análoga a lo que sucede con la biomasa aérea. Por ejemplo, los manglares enanos son bosques dominados por $R$. mangle, y aunque presentan una gran densidad de individuos, su bajo porte (altura menor a $1.5 \mathrm{~m}$ ) genera que éstos sean los bosques con menor contenido de carbono. Por otro lado, los manglares oceánicos del Indo-Pacífico presentan gran variación en composición y altura, incluyendo bosques con altura inferior a $4 \mathrm{~m}$, hasta zonas con mangles de alturas superiores a $15 \mathrm{~m}$. Sin embargo, aproximadamente la mitad de los sitios muestreados para la estimación de biomasa correspondían a bosques altos (altura mayor de 15 m) (Donato et al., 2011). Adicionalmente, las estimaciones de biomasa aérea y carbono en el Indo-Pacífico se realizaron a partir ecuaciones alométricas publicadas, lo cual puede llevar a sobre o subestimaciones. En el caso del presente estudio, el contenido de carbono encontrado para los bosques de manglar del DMI Cispatá en la biomasa aérea $(64.85 \pm 10.12 \mathrm{Mg} \mathrm{C} / \mathrm{ha})$, estuvo entre los valores promedio reportados para los bosques de manglar a nivel mundial, y fue similar a los valores reportados para los bosques altos dominados por $R$. mangle en la península de Yucatán, México (69.6 Mg/ha) (Adame et al., 2013).

La comprensión de la variación espacial en el almacenamiento de carbono en los hábitats naturales es fundamental para los esfuerzos de mitigación del cambio climático como la Reducción de Emisiones por Deforestación y Degradación (REDD+) (Sierra et al., 2007; Köhl, Lister, Scott, Baldauf, \& Plugge, 2011; Hutchison, Manica1, Swetnam, Balmford, \& Spalding, 2013). Sin embargo, la distribución del "Blue Carbon" o carbono azul como es conocido el carbono que se almacena en marismas, pastos marinos y manglares, sigue siendo poco conocida, en comparación con los grandes avances que se ha tenido en las últimas décadas en los ecosistemas forestales terrestres (Hutchison et al., 2013). De acuerdo con el mapa global sobre el potencial de almacenamiento de carbono en la biomasa aérea de los manglares (Hutchison et al., 2013), Colombia es uno de los 10 países que más potencial de almacenamiento de carbono tiene en sus manglares, dado que su biomasa aérea promedio es de $253.2 \mathrm{Mg} / \mathrm{ha}$. El promedio obtenido para los manglares del DIM de Cispatá, corresponde al $51 \%$ de dicho valor. Estos resultados sugieren que Colombia tiene alto potencial para participar en la conservación de estos ecosistemas, y reportar grandes beneficios por unidad de área. Adicionalmente, y acorde con las recomendaciones de Hutchison et al., (2013), la aplicación efectiva de políticas de manejo y conservación de bosques a nivel nacional, podría hacer una importante contribución a los flujos globales de carbono, y a la contribución nacional del sector AFOLU (Agriculture, Forestry and Other Land Use por sus siglas en inglés) en el futuro acuerdo Post-Kioto previsto para 2015.

\section{AGRADECIMIENTOS}

Los autores agradecen al Departamento Administrativo de Ciencia, Tecnología e Innovación - Colciencias por todo el apoyo brindado 
a través de sus Planes de Fortalecimiento Institucional 2009-2012. Al FMAM, el PNUD, INVEMAR-MADS, y CVS por su financiación a través del proyecto COL-00075241, PIMS \# 3997, proyecto de implementación nacional (NIM) y con contrapartida del banco de proyectos de inversión de la Nación. A la Fundación Natura por su apoyo a través de la iniciativa MVC Colombia, cofinanciada por el GEF a través del BID. A la Universidad Nacional de Colombia - Sede Medellín por toda la colaboración logística para la realización de las fases de campo y laboratorio. A la comunidad de Bahía de Cispatá por su acogida y acompañamiento durante la fase de campo, y al personal administrativo y científico de Carbono \& Bosques por su constante colaboración.

\section{RESUMEN}

La distribución de carbono azul en los ecosistemas, entre los que se cuentan los manglares, es poco conocida en comparación con los grandes avances que existen para los ecosistemas forestales terrestres, a pesar de que su alta productividad y capacidad de almacenamiento de carbono es reconocida. Este estudio, tuvo por objetivo, analizar la biomasa aérea de las especies Rhizophora mangle y Avicennia germinans en el ecosistema de manglar localizado en el área marina protegida denominada Distrito de Manejo Integrado (DMI) Cispatá-Tinajones-La Balsa en el Caribe colombiano. Para ello, con la debida autorización oficial, se cosecharon 30 individuos de cada especie, con el fin de generar modelos alométricos que permitieran estimaciones de biomasa aérea con bajos niveles de incertidumbre. Los resultados indican que la biomasa aérea de los bosques de manglar del Caribe colombiano en el DMI, es de $129.69 \pm$ $20.24 \mathrm{Mg} / \mathrm{ha}$, es decir, que almacenan $64.85 \pm 10.12 \mathrm{MgC} /$ ha. Teniendo en cuenta que el DMI presenta un área de 8 570.9 ha en bosques de manglar, se estima que el carbono total potencialmente almacenado por este ecosistema es de aproximadamente $555795.93 \mathrm{MgC}$. Las ecuaciones generadas en este estudio se pueden considerar como una opción alternativa para la evaluación de las reservas de carbono en la biomasa aérea de los bosques de manglar en del Caribe colombiano, dado que otros modelos disponibles para la estimación de la biomasa aérea no discriminan a los bosques de manglar, a pesar de ser ecosistemas particulares. Los modelos pueden ser empleados como una opción para apoyar las estrategias de conservación de bosques y la reducción de emisiones. En general, el potencial de almacenamiento de carbono en los bosques de manglar del Caribe colombiano, es importante, y podría posesionar al país en la conservación de bosques y la reducción de emisiones a través de la conservación del carbono azul almacenado.

Palabras clave: A. germinans, biomasa aérea, carbono, ecuaciones alométricas, manglar, $R$. mangle.

\section{REFERENCIAS}

Achard, F., Defries, R., Eva, H., Hansen, M., Mayaux, P., \& Stibig, H. J. (2007). Pantropical monitoring of deforestation. Environmental Research Letters, 2, 1-11.

Adame, M. F., Kauffman, J. B., Medina, I., Gamboa, J. N., Torres, O., Caamal, J. P., Reza, M., \& HerreraSilveira, J. A. (2013). Carbon Stocks of Tropical Coastal Wetlands within the Karstic Landscape of the Mexican Caribbean. PLoS One, 8(2), e56569. doi:10.1371/journal.pone.0056569.

Agudelo, C. M., Bolívar, J., Polanía, J., Urrego, L. E., Yepes, A., \& Sierra, A. (2014). Estructura y composición florística de los manglares de la Bahía de Cispatá (Caribe colombiano). Informe Técnico, Carbono \& Bosques. Medellín.

Alongi, D. M. (2002). Present state and future of the world's mangrove forests. Environmental Conservation, 29, 331-349.

Alongi, D. M. (2009). The Energetics of Mangrove Forests. Dordrecht, Springer.

Álvarez, E., Duque, A., Saldarriaga, J. G., Cabrera, K., De las Salas, G., Del Valle, J. I., Moreno, F., Orrego, S. A., \& Rodríguez, L. (2012). Tree above-ground biomass allometries for carbon stocks estimation in the natural forests of Colombia. Forest Ecology and Management, 267, 297-308.

Aragão, L., Malhi, Y., Metcalfe, D. B., Silva-Espejo, J. E., Jiménez, E., Navarrete, D., Almeida, S., Costa, A. C. L., Salinas, N., Phillips, O. L., Anderson, L. O., Álvarez, E., Baker, T. R., Goncalvez, P. H., Huamán-Ovalle, J., Mamani Solórzano, M., Meir, P., Monteagudo, A., Patiño, S., Peñuela, M. C., Prieto, A., Quesada, C. A., Rozas Dávila, A., Rudas, A., Silva Jr., J. A., \& Vásquez, R. (2009). Above- and below-ground net primary productivity across ten Amazonian forests on contrasting soils. Biogeosciences, 6, 2759-2778.

Baker, T. R., Phillips, O. L., Malhi, Y., Almeida, S., Arroyo, L., Di Fiore, A., Erwin, T., Killeen, T. J., Laurance, S. G., Laurance, W. F., Lewis, S. L., Lloyd, J., Monteagudo, A., Neill, D. A., Patino, S., Pitman, N. C .A., Silva, M., \& Vasquez- Martinez, R. (2004). Variation in wood density determines spatial patterns in Amazonian forest biomass. Global Change Biology, 10, 545-562.

Brown, S. (1997). Estimating biomass and biomass change of tropical forests: A primer. Food and Agriculture Organization, Roma. FAO Forestry Paper no. 134. 
Brown, B., Hall, M., Andrasko, K., Ruíz, F., Marzoli, W., Guerrero, G., Masera, O., Dushku, A., Dejong, B., \& Cornell, J. (2007). Baselines for land-use change in the tropics: application to avoided deforestation projects. Mitigation and Adaptation Strategies for Global Change, 12, 1001-1026.

Canavos, G. C. (1988). Probabilidad y estadística. Aplicaciones y métodos. Virginia common wealth University. México: McGraw-Hill.

Chave, J., Andalo, C., Brown, S., Cairns, A., Chambers, J. Q., Folster, H., Fromard, F., Higuchi, N., Kira, T., Lescure, J. P., Nelson, B. W., Ogawa, H., Puig, H., Riera, B., \& Yamakura, T. (2005). Tree allometry and improved estimation of carbon stocks and balance in tropical forests. Oecologia, 145, 87-9.

Clark, D. A. (2007). Detecting tropical forests responses to global climatic and atmospheric change: current challenges and a way forward. Biotropica, 39(1), 4-19.

Clark, D. A., Brown, S., Kicklighter, D. W., Chambers, J. Q., Thomlinsom, J. R., Ni, J., \& Holland, E. A. (2001). Net primary production in tropical forest: an evaluation and synthesis of existing data. Ecological Applications, 11, 371-384.

Cochran, W. (2000). Técnicas del muestreo. México: Compañía Editorial Continental.

Corporación Autónoma Regional de los Valles del Sinú y del San Jorge (CVS), Corporación Nacional de Investigación y Fomento Forestal (CONIF), \& Organización Internacional de Maderas (OIMIT). (2005). Plan de manejo integral de los Manglares de la Zona de Uso Sostenible del sector Estuarino de la Bahía de Cispatá, Departamento de Córdoba.

Corporación Autónoma Regional de los Valles del Sinú y del San Jorge (CVS), \& Instituto Alexander von Humboldt (IAvH). (2006). Delimitación y formulación de un distrito de Manejo Integrado de los Recursos naturales (DMI) de los manglares de la bahía de Cispatá, Tinajones, La Balsa y sectores aledaños ( $p$. 299). Montería, Colombia: Corporación Autónoma Regional de los Valles del Sinú \& San Jorge (CVS)Instituto Alexander von Humboldt (IAvH), convenio 026.

Corporación Autónoma Regional de los Valles del Sinú y del San Jorge (CVS), \& Instituto de Investigaciones Marinas y Costeras (INVEMAR). (2010). Plan Integral de Manejo Distrito de Manejo Integrado (DMI) bahía de Cispatá - La Balsa - Tinajones y sectores aledaños. En G. X. Rojas y P. C. Sierra-Correa (Eds.) Serie de Publicaciones Especiales No. 18 de INVEMAR. Santa Marta.

Donato, D. C., Kauffman, B., Murdiyarso, D., Kurnianto, S., Stidham, M., \& Kanninen, M. (2011). Mangroves among the most carbon-rich forests in the tropics. Nature Geoscience, 4, 293-297.
Duke, N. C., Meynecke, J. O., Dittmann, S., Ellison, A. M., Anger, K., Berger, U., Cannicci, S., Diele, K., Ewel, K. C., Field, C. D., Koedam, N., Lee, S. Y., Marchand, C., Nordhaus, I., \& Dahdouh-Guebas, F. (2007). A World Without Mangroves? Science, 317 (5834), 41-42.

Ewel, K. C., Bourgeois, J. A., Cole, T. G., \& Zheng, S. (1998). Variation in environmental characteristics and vegetation in high-rainfall mangrove forests, Kosrae Micronesia. Global Ecology and Biogeography Letters, 7, 49-56.

Fatoyinbo, T. E., \& Simard, M. (2013). Height and biomass of mangroves in Africa from ICESat/GLAS and SRTM, International Journal of Remote Sensing, 34(2), 668-681.

Fatoyinbo, T. E., Simard, M., Washington-Allen, R. A., \& Shugart, H. H. (2008). Landscape-scale extent, height, biomass, and carbon estimation of Mozambique's mangrove forests with Landsat ETM+ and Shuttle Radar Topography Mission elevation data. Journal of Geophysical Research, 113, G02S06, 1-13.

Fearnside, P. M., Lashof, D. A., \& Moura-Costa, P. (1999). Accounting for time in Mitigating Global Warming through land-use change and forestry. Mitigation and Adaptation Strategies for Global Change, 5(3), 239-270.

Fearnside, P. M., \& Laurance, W. F. (2004). Tropical deforestation and greenhouse gas emissions. Ecological Applications, 14, 982-6.

Fromard, F., Puig, H., Mougin, E., Marty, G., Betoulle, J. L., \& Cadamuro, L. (1998). Structure, above groundbiomass and dynamics of mangrove ecosystems: new data from French Guiana. Oecologia, 115(1), 39-53.

Gibbs, H. K., Brown, B., Niles, J. O., \& Foley, J. A. (2007). Monitoring and estimating tropical forest carbon stocks: making REDD a reality. Environmental Research Letters, 2, 1-13.

Gofc-Gold. (2009). Reducing greenhouse gas emissions from deforestation and degradation in developing countries: a sourcebook of methods and procedures for monitoring, measuring and reporting (GOFCGOLD Report version COP14-2). Alberta, Canada: GOFC-GOLD Project Office, Natural Resources Canada.

Holdridge, L. R., Grenke, W., Hatheway, W. H., Liang, T., \& Tosi, J. A. (1971). Forest Environments in Tropical Life Zones: A Pilot Study. Oxford, New York: Pergamon Press.

Houghton, R. A. (2005). Tropical deforestation as a source of greenhouse gas emissions. In P. Mutinho \& S. Schwartzman (Eds.), Tropical Deforestation and Climate Change. Belem: IPAM. 
Hutchison, J., Manica, A., Swetnam, R., Balmford, A., \& Spalding, M. (2013). Predicting Global Patterns in Mangrove Forest Biomass. Conservation Letters, 7(3), 233-240.

Intergovernmental Panel on Climate Change (IPCC). 2007. Climate change: the physical science basis. Cambridge, UK: Cambridge University Press.

Jennerjahn, T. C., \& Ittekkot, V. (2002). Relevance of mangroves for the production and deposition of organic matter along tropical continental margins. Naturwissenschaften, 89, 23-30.

Köhl, M., Lister, A., Scott, C., Baldauf, T., \& Plugge, D. (2011). Implications of sampling design and sample size for national carbon accounting systems. Carbon Balance and Management, 6, 10.

Komiyama, A., Moriya, H., Prawiroatmodjo, S., Toma, T., \& Ogino, K. (1988), Primary productivity of mangrove forest, Biologial System of Mangrove (A Report of East Indonesian Mangrove Expedition 1986, 97-117). Ehime: Ehime Univ.

Lacher, W. (1977). Ecofisiologia vegetal. España: Omega.

Lema, A. (2003). Elementos Estadísticos de Dasometría y Medición Forestal. Medellín, Colombia: SILVANO Ltda.

Lovelock, C. E., Ruess, R. W., \& Feller, I. C. (2011). $\mathrm{CO}_{2}$ efflux from cleared mangrove peat. PloS one, 6, e21279.

Lugo, A. E., \& Snedaker, S. C. (1974). The Ecology of Mangroves. Annual Review of Ecology and Systematics, 5(1), 39-64.

Malhi, Y., Aragão, L. E. O. C., Metcalfe, D. B., Paiva, R., Quesada, C. A., Almeida, A., Anderson, L., Brando, P., Chambers, J. Q., Da Costa, A. C. L., Hutyra, L. R., Oliveira, P., Patiño, S., Pyle, E. H., Robertson, A. L., \& Texeira, L. M. (2009). Comprehensive assessment of carbon productivity, allocation and storage in three Amazonian forests. Global Change Biology, 1-20.

Malhi, Y., \& Phillips, O. L. (2004). Tropical forests and global atmospheric change: a synthesis. Philosophical Transactions of the Royal Society B: Biological Sciences, 359, 549-555.

McDicken, K. G. (1997). A guide to monitoring carbon storage in forestry and agroforestry projects. Virginia, USA: Winrock International Institute for Agricultural Development, Forest Carbon Monitoring Program.

Navar, J. (2009). Biomass component equations for Latin American species and groups of species. Annals of Forest Science, 66, 208.

Olander, L. P., Gibbs, H. K., Steininger, M., Swenson, J. J., \& Murray, B. C. (2008). Reference scenarios for deforestation and forest degradation in support of
REDD: a review of data and methods. Environmental Research Letters, 3, 025011.1-11.

Ong, J. E., Gong, W. K., \& Wong, C. H. (1981). Ecological Monitoring of the Sungai Merbok Estuarine Mangrove Ecosystem. Penang: Universiti Sains Malaysia.

Picard, N., Saint-André, L., \& Henry, M. (2012). Manual for building tree volume and biomass allometric equations: from field measurement to prediction. Food and Agricultural Organization of the United Nations, Rome, and Centre de Coopération Internationale en Recherche Agronomique pour le Développement, Montpellier.

R Development Core Team. (2010). R: A Language and Environment for Statistical Computing. Version 2.9.0 Patched. The R Foundation for Statistical Computing. Vienna, Austria. Recuperado de http://www.Rproject.org

Ray, R., Ganguly, D., Chowdhury, C., Dey, M., Das, S., Dutta, M. K., Mandal, S. K., Majumder, N., De, T. K., Mukhopadhyay, S. K., \& Jana, T. K. (2010). Carbon sequestration and annual increase of carbon stock in a mangrove forest. Atmospheric Environment, 45(2011), 5016-5024.

Rivera-Monroy, V. H, Twilley, R., Medina, E., Moser, E. B., Botero, L., Francisco, A.M., \& Bullard, E. (2004). Spatial variability of soil nutrients in disturbed riverine mangrove forests at different stages of regeneration in the San Juan River Estuary, Venezuela. Estuaries, 27, 44-57.

Robertson, K., \& Chaparro, J. (1998). Evolución histórica del río Sinú. Cuadernos de Geografia, 7(1-2), 70-87.

Rügnitz, M. T., Chacón, M. L., \& Porro, R. (2009). Guía para la Determinación de Carbono en Pequeñas Propiedades Rurales (Manual Técnico No. 11). Lima, Perú: Centro Mundial Agroflorestal (ICRAF)/Consórcio Iniciativa Amazônica (IA).

Saenger, P., \& Snedaker, S. C. (1993), Pantropical trends in mangrove aboveground biomass and annual litterfall. Oecologia, 96(3), 293-299.

Sánchez-Páez, H., Ulloa-Delgado, G., \& Tavera-Escobar, T. (2004). Manejo integral de manglares por сотиnidades locales, Caribe de Colombia (p. 335). Ministerio de Ambiente, Vivienda y Desarrollo Territorial. CONIF-OIMT.

Sánchez-Páez, H., Ulloa-Delgado, G., Tavera Escobar, H., \& Gil Torres, W. (2005). Plan de manejo integral de los manglares de la zona de uso sostenible del sector estuarino de la Bahía de Cispatá departamento de Córdoba - Colombia (p. 202). Bogotá, D.C.: OIMT, CVS, CONIF, Ministerio de Ambiente, Vivienda y Desarrollo Territorial.

Sierra, C. A., Del Valle, J. I., Orrego, S. A., Moreno, F. H., Harmon, M. E., Zapata, M., Colorado, G. J., Herrera, 
M. A., Lara, W., Restrepo, D. E., Berrouet, L. M., Loaiza, L. M., \& Benjumea, J. F. (2007). Total carbon stocks in a tropical forest landscape of the Porce region, Colombia. Forest Ecology and Management, 243, 299-309.

Soares, M. L. G., \& Schaeffer-Novelli, Y. (2005). Aboveground biomass of mangrove species. I. Analysis of models. Estuarine, Coastal and Shelf Science, 65(1-2), 1-18.

Spaulding, M. D., Blasco, F., \& Field, C. D. (1997). World Mangrove Atlas. Okinawa, Japan: International Society for Mangrove Ecosystem.

Walters, B. B., Rönnbäck, P., Kovacs, J., Crona, B., Hussain, S., Badola, R., Primavera, J., Barbier, E. B., \& Dahdouh-Guebas, F. (2008). Ethnobiology, socio-economics and adaptive management of mangroves: A review. Aquatic Botany, 89, 220-236.

Yepes, A. P., Navarrete, D. A., Duque, A. J., Phillips, J. F., Cabrera, K. R., Álvarez, E., García, M. C., Ordoñez, M. F. (2011). Protocolo para la estimación nacional y subnacional de biomasa - carbono en Colombia. Bogotá D.C., Colombia: Instituto de Hidrología, Meteorología, y Estudios 690 Ambientales-IDEAM.

West, G. B., Brown, J. H., \& Enquist, B. J. (1999). A general model for the structure and allometry of plant vascular systems. Nature, 400, 664-667.

Zianis, D. (2008). Predicting mean aboveground forest biomass and its associated variance. Forest Ecology and Management, 256(6), 1400-1407. 